\title{
Evaluation of Matrix-Assisted Laser Desorption Ionization-Time-of-Flight Mass Measurement Accuracy by Using Delayed Extraction
}

\author{
Ricky D. Edmondson and David H. Russell \\ Laboratory for Biological Mass Spectrometry, Department of Chemistry, Texas A \& M University, College \\ Station, Texas, USA
}

Mass measurement accuracy of a reflectron time-of-flight mass spectrometer equipped with delayed extraction is evaluated. Mass resolution of $10,000-15,000$ is achieved routinely for peptides in the mass range of 1-6 ku ionized by matrix-assisted laser desorption ionization. The mass measurement accuracy of peptides with molecular weights of $1-4 \mathrm{ku}$ is $10-15 \mathrm{ppm}$ by using external calibration and better than $5 \mathrm{ppm}$ by using internal calibration. The mass calibration remains accurate over a broad mass-to-charge ratio range (1-4 ku), even when the calibration curve is extrapolated several thousand mass units. (J Am Soc Mass Spectrom 1996, 7, 995-1001)

$O^{1}$ ince the introduction of matrix-assisted laser desorption ionization (MALDI) by Karas and Hillenkamp [1], time-of-flight (TOF) has evolved rapidly as a routine analytical mass spectrometer for the analysis of biomolecules. The analytical advantages of TOF include its relatively low cost, high sensitivity, large mass range (in excess of $100 \mathrm{ku}$ ), and the ability to record a complete spectrum in a single acquisition. The major disadvantage of TOF is low resolution. The mass resolution of MALDI-TOF is especially poor because the ions are formed with a broad kinetic energy distribution [2] and a mass-independent initial velocity $[3,4]$. The mass resolution of TOF and MALDI-TOF can be improved by incorporation of an ion reflector [5], but metastable ion decay in the field-free region can skew the peak shapes [6]. Because isotope peaks rarely are resolved in MALDI mass spectra, mass calibration is performed by using some method to peakcentroid a broad unresolved ion signal. Mass calibration via peak-centroiding methods gives reasonably good mass measurement accuracy; for example, Beavis and Chait [7] reported mass accuracies of $0.01 \%$ (100 ppm) for MALDI-TOF. An obvious limitation of peak-centroiding arises if the peak is skewed due to unresolved ions. For example, many proteins are highly heterogeneous due to phosphorylation, glycosylation, or other types of covalent modifications. In addition, MALDI can produce multiple ions of the analyte, for example, $[\mathrm{M}+\mathrm{H}]^{+},[\mathrm{M}+\mathrm{Na}]^{+}$, and $[\mathrm{M}+\text { matrix }]^{+}$ adduct ions. These ions may not be resolved in the MALDI-TOF mass spectrum. The presence of these

Address reprint requests to Dr. David H. Russell, Director, Laboratory for Biological Mass Spectrometry, Department of Chemistry, Texas A \& M University, College Station, TX 77843. ions results in a higher measured mass for the analyte. It is also possible that fragmentation of the analyte (especially loss of $\mathrm{H}_{2} \mathrm{O}, \mathrm{CO}_{2}$, or other small neutral fragments) reduces the measured mass [8]. Even when artificial peak-broadening does not exist, for example, the width of the peak is the same as the isotopic envelope, and individual isotopes are not resolved, there are problems with accurate $(<50-\mathrm{ppm})$ mass calibration [9].

The first significant improvement in TOF mass resolution was achieved by incorporatiog of time-lag focusing (TLF) [10]. Several researchers have discussed the principles of TLF or delayed extraction (DE) as it relates to MALDI-formed ions and have shown its use to improve resolution significantly [11-14]. DE involves ion formation in a field-free region and application of a high voltage pulse to accelerate the ions from the source a few hundred nanoseconds after ion formation. On the basis of the previous experimental work, it is clear that $\mathrm{DE}$ permits mass resolution in excess of 10,000 for analytes that have mass-to-charge ratios of 1-6 ku. The ability to resolve isotope peaks fully for peptides under $6000 \mathrm{u}$ should improve the mass measurement accuracy of MALDI greatly. An increase in resolution does not necessitate a corresponding increase in mass measurement accuracy. For example, mass resolution of $>100,000$ has been demonstrated for biomolecules by using MALDI-Fourier transform ion cyclotron resonance; however, frequency shifts due to imperfect $\mathrm{E} \times \mathrm{B}$ fields complicate mass calibration $[15,16]$.

Mass measurement accuracy values for MALDIformed ions via delayed extraction on linear TOF instruments were reported by Whittal and $\mathrm{Li}$ [14] and Brown and Lennon [17]. By using internal calibrants, 
Whittal and $\mathrm{Li}$ reported mass measurement accuracies for peptides of 5-70 ppm with average values of 30$50 \mathrm{ppm}$, and Brown and Lennon reported mass measurement accuracies of $30-50 \mathrm{ppm}$. In this article we evaluate the mass measurement accuracy of MALDITOF by using a reflectron instrument equipped with delayed extraction. The purpose of this study is to determine the effect of delayed extraction, in particular delay times and pulse voltages, on MALDI-TOF mass measurement accuracy. For this study 'we chose analytes with molecular weights of $1000-4000 \mathrm{u}$ because these analytes give an appreciable abundance for the ${ }^{12} \mathrm{C}$ isotope peak. The ${ }^{12} \mathrm{C}$ isotope peak is used for mass calibration to minimize errors associated with variation of isotopic abundances $[18,19]$.

\section{Experimental}

The experiments were performed by using a PerSeptive Biosystems Voyager Elite XL time-of-flight mass spectrometer. A detailed description of this instrument was given by Vestal et al. [13]. Briefly, a pulsed nitrogen laser is used to form ions in a gridded two-stage continuous or pulsed (delayed extraction) ion source. In the continuous mode, ions are formed under a high electric field and are immediately accelerated into the field-free drift tube. With delayed extraction, the ions are formed with the repeller and first grid at the same potential. After a delay of a few hundred nanoseconds, the repeller is switched to a higher potential. The voltage difference between the repeller and the first grid is referred to as the pulse voltage. The ions are accelerated into a 4.2-m flight tube. A negatively biased wire ion guide (WIG) [20] spans the full length of the field-free region $(\sim 3.3 \mathrm{~m})$; it extends from the source exit to the entrance of the single-stage, gridded reflectron $(\sim 0.9 \mathrm{~m})$. The WIG potential is continuously variable; typically the wire is biased at $-1 \mathrm{~V}$. The effective reflected flight path is $\sim 6.6 \mathrm{~m}$. The reflected ions are detected by a coaxial dual microchannel plate detector. The ion signal is recorded by using a 500-MHz Precision Instruments digitizer board at the rate of $500 \mathrm{MS} / \mathrm{s}$ and then processed and stored by using standard Voyager software.

All spectra shown in this article were acquired with a laser fluence of $15 \mathrm{~mJ} / \mathrm{cm}^{2}$, a laser repetition rate of $3 \mathrm{~Hz}$, and a total acceleration of $20 \mathrm{kV}$. The pulse voltage and delay time used to acquire the reported data were $6 \mathrm{kV}$ and $150 \mathrm{~ns}$ unless otherwise noted. The pressure of the source region and analyzer were $6 \times$ $10^{-8}$ torr and $5 \times 10^{-9}$ torr, respectively.

The mass values for the peptides listed in this article are for monoisotopic values. The mass assignment for each peptide was performed by first centroiding the ${ }^{12} \mathrm{C}$ isotope peak in time. The centroid was calculated from the top $50 \%$ of the peak by using standard Voyager software. From repetitive measurements we estimate the reproducibility in centroiding a single peak is \pm 10 ps. For example, 20 successive centroids of a single peak differed by no more than $10 \mathrm{ps}$. The factors that effect the reproducibility of the measured flight times of a peak from spectra to spectra include the number of data points across the peak, surface imperfections of the sample, drift in the high voltage power supplies, and the jitter associated with the start trigger and high voltage switch. These factors, as well as their effect on measured flight times, are discussed herein.

The TOF mass calibration equation (eq 1) was used to calibrate all the spectra'shown. The calibration constants $\left(A_{1}, A_{2}\right)$ of eq 1 were determined by using the flight times of two components with known mass-tocharge ratio values. Mass assignment was then made based on the flight time of the analyte ions.

$$
t=A_{1}(m / z)^{1 / 2}+A_{2}
$$

\section{Sample Preparation}

Sample spots were prepared on a multistage sample plate similar to the one described by Vestal et al. [13]. Because ion yields and flight times for the etched sample wells on the standard multistage plate are erratic, we prefer to use a smooth plate. The sample plate used has a smooth stainless steel surface that was polished with 12,000 grit sandpaper. The matrices $\alpha$ cyano-4-hydroxycinnamic acid (ACCA) and sinapic acid (SA) were obtained from Aldrich Chemical Co. (Milwaukee, WI) and were used without further $\mathrm{pu}$ rification. The peptides were obtained from Sigma Chemical Co. (St. Louis, MO).

The sample spots were prepared by using an overlayer method that produces homogeneous surfaces and minimizes so-called hot spots. A matrix solution was prepared by dissolving $35 \mathrm{mg}$ of ACCA in $1 \mathrm{~mL}$ of methanol. A matrix bed was prepared by applying $0.5 \mu \mathrm{L}$ of this solution to the sample plate and allowing it to dry. The matrix-analyte solutions were prepared with 2:1 water:methanol that had final matrix and analyte concentrations of $10 \mathrm{mM}$ and 1-3 $\mu \mathrm{M}$, respectively. A small amount $(\sim 0.3 \mu \mathrm{L})$ of the matrix-analyte solution was applied to the matrix bed and was allowed to air dry. The small amount of solution does not redissolve the matrix bed. The dried sample was then washed with $10 \mu \mathrm{L}$ of deionized water, as described by Beavis and Chait [7]. All of the spectra shown, with the exception of Figure 5, were acquired from sample spots where ACCA was used as the matrix in both the matrix-analyte solution as well as the matrix bed. Figure 5 was acquired by using SA in the matrix-analyte solution, while the matrix bed consisted of only ACCA.

\section{Results}

In this article we present the results of studies designed to evaluate the mass measurement accuracy of 

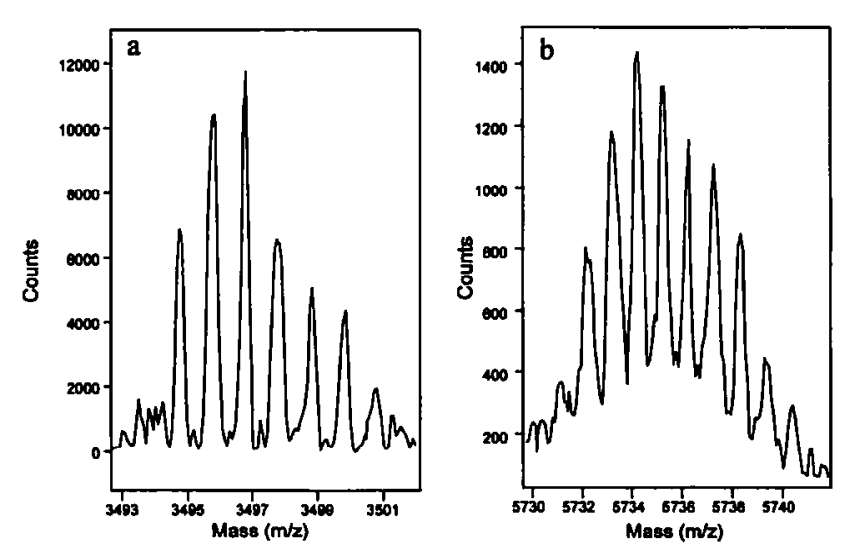

Figure 1. High resolution MALDI/DE-RTOF mass spectra of (a) bovine insulin b-chain ( $m / \Delta m=15,000)$ acquired by using a total acceleration of $25 \mathrm{kV}$, a pulse voltage of $7 \mathrm{kV}$, and a $300-\mathrm{ns}$ delay time and (b) bovine insulin $(m / \Delta m=12,500)$ acquired at a total acceleration of $25 \mathrm{kV}$, a pulse voltage of $9 \mathrm{kV}$, and a 275-ns delay time.

MALDI-reflectron time-of-flight (MALDI-RTOF) equipped with delayed extraction (MALDI/DE-RTOF). Both internal and external calibrations are evaluated by using peptides with molecular weights between 1046 and $3495 \mathrm{u}$. In addition, the dependence of the mass measurement accuracy on the pulse voltages and delay times are examined. Figure 1 contains MALDI/DE-RTOF mass spectra of bovine insulin bchain and bovine insulin. Figure 2 contains the MALDI/DE-RTOF mass spectrum of a synthetic peptide acquired with a total acceleration of $20 \mathrm{kV}$ by using a pulse voltage of $6 \mathrm{kV}$ and a delay of $300 \mathrm{~ns}$. These mass spectra illustrate the resolution that can be obtained by MALDI/DE-RTOF. It is our experience that mass resolution $(m / \Delta m)$ greater than 10,000 is routine on the Voyager Elite $\mathrm{XL}$ when the sample preparation method described herein is used, and the

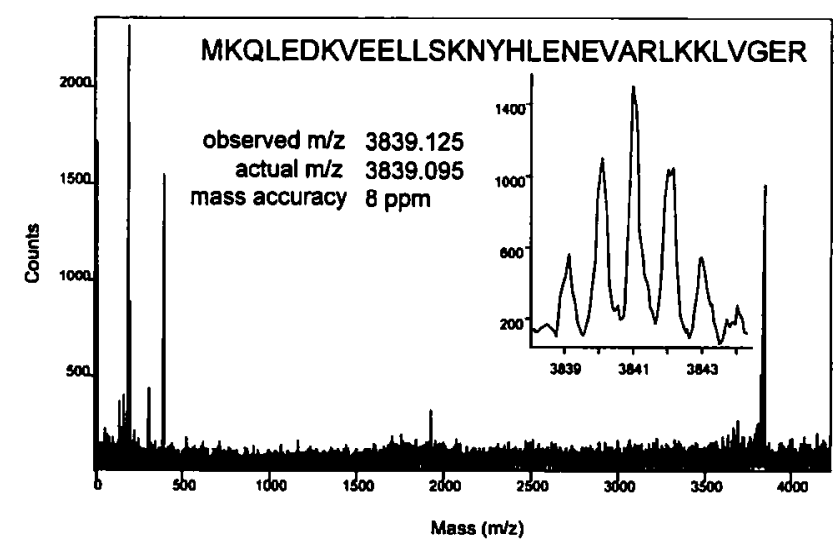

Figure 2. High resolution MALDI/DE-RTOF mass spectrum of a synthetic peptide $(m / \Delta m=12,500)$ acquired with total acceleration of $20 \mathrm{kV}$, a pulse voltage of $6 \mathrm{kV}$, and a 300-ns delay time. The amino acid sequence of the peptide is given as the single letter designation. External calibration yields mass measurement accuracy of $8 \mathrm{ppm}$.

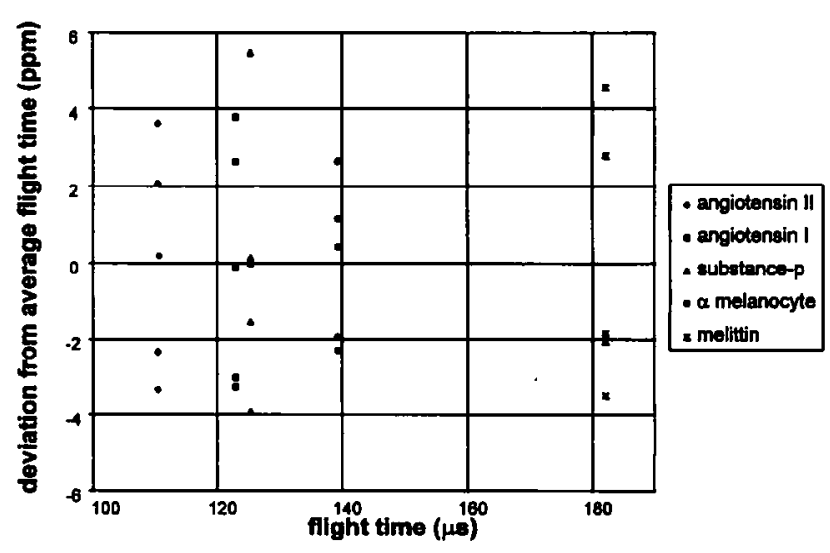

Figure 3. A plot of the variation in the flight time (expressed as parts per million) of analyte ions taken from a single -sample spot.

remainder of the article will focus on the issue of mass measurement accuracy.

Figures 3 and 4 contain data on the flight time reproducibility of ions generated by MALDI/DERTOF. Flight time reproducibility of MALDI/DE-RTOF spectra taken from a single sample spot are compared in Figure 3. Separate sample spots were prepared for angiotensin I $\left(M_{\mathrm{r}}=1295.677\right)$, angiotensin II $\left(M_{\mathrm{r}}=1045.534\right)$, substance $\mathrm{P}\left(M_{\mathrm{r}}=1346.728\right)$, $\alpha$ melanocyte-stimulating hormone $\left(M_{\mathrm{r}}=1663.793\right)$, and melittin $\left(M_{\mathrm{r}}=2844.754\right)$. Five spectra, which each consisted of 50 averaged laser shots, were taken from each sample spot at a mass resolution of $\sim 6500$. The data points in Figure 3 represent the deviation, expressed in parts per million, of each flight time from the average flight time for that particular analyte.

Deviation $(\mathrm{ppm})=[($ flight time - avg. flight time $) /$

(avg. flight time) $] \times 10^{6}$

Flight time reproducibility of MALDI/DE-RTOF spectra taken from different sample spots is compared in Figure 4. Six sample spots were made near the center of the sample plate; each spot contained three analytes:

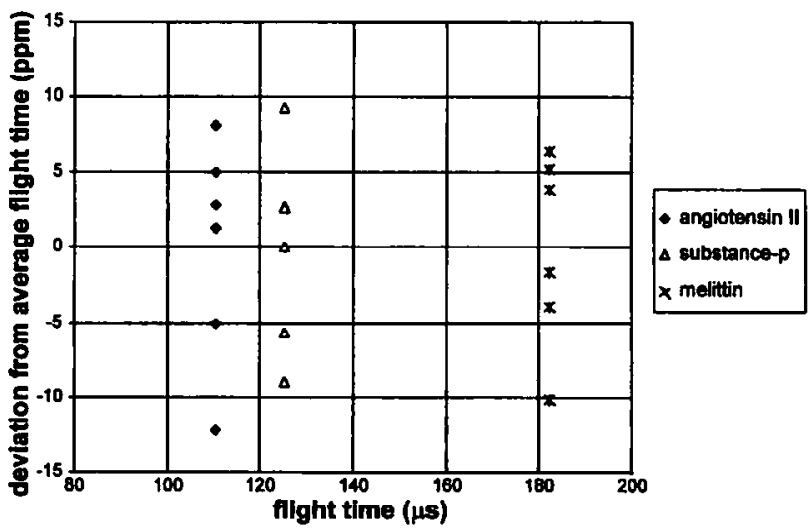

Figure 4. A plot of the variation in the flight time (expressed as parts per million) of analyte ions taken from six different sample spots. 
Table 1. Mass accuracy of MALDI/DE-RTOF with external calibration

\begin{tabular}{|c|c|c|c|c|c|}
\hline Compound & $\begin{array}{c}\text { Actual } \\
m / z\end{array}$ & $\begin{array}{c}\text { Observed } \\
m / z\end{array}$ & $\Delta m / z$ & $\begin{array}{c}\text { Mass } \\
\text { accuracy } \\
\text { (ppm) }\end{array}$ & $\begin{array}{c}\text { Average } \\
\text { mass } \\
\text { accuracy } \\
\text { (ppm) }\end{array}$ \\
\hline Angiotensin II & 1046.542 & 1046.557 & 0.015 & 14 & \\
\hline Angiotensin I & 1296.685 & 1296.674 & -0.011 & -9 & \\
\hline Substance $P$ & 1347.736 & 1357.714 & -0.022 & -16 & \\
\hline \multirow[t]{2}{*}{$\alpha$-Melanocyte } & 1664.801 & 1664.821 & 0.020 & 12 & \\
\hline & & & & & 13 \\
\hline
\end{tabular}

angiotensin $I$, substance $P$, and melittin. A spectrum that consisted of an average of 50 laser shots was taken at each sample spot. The deviation (in parts per million) in flight time from the average flight time for each analyte is plotted in Figure 4.

Tables 1 and 2 contain data on the mass accuracy of MALDI/DE-RTOF for the use of external mass calibration and internal mass calibration. Table I contains data from MALDI/DE-RTOF spectra obtained from sample spots that contain individual analytes. Five spectra, which consist of 50 averaged laser shots, were taken from each sample spot, and the average flight time for each analyte was determined. A separate sample spot that contained melittin and ACCA was used to generate a mass calibration curve by using eq 1 . This calibration was applied to the average flight time for each analyte and the mass-to-charge ratio value and mass accuracy were calculated. For the data shown in Table 2, a sample spot was prepared that contained $\alpha$-melanocyte, melittin, and bovine insulin b-chain. The resolution of each analyte was $\sim 6500$. Ten spectra were acquired; each consisted of 100 laser shots. A calibration curve was generated for each spectra by using the $\alpha$-melanocyte $[\mathrm{M}+\mathrm{H}]^{+}$ion and the ACCA $[2 \mathrm{M}+\mathrm{H}]^{+}$ion. The measured mass-to-charge ratio value for each spectra, as well as the average mass-to-charge ratio value, is listed in the table.

A detailed evaluation of flight time reproducibility and peak centroiding was performed by using the $[\mathrm{M}+\mathrm{H}]^{+}$ions of bovine insulin b-chain. A pulse voltage of $6 \mathrm{kV}$ and a delay time of $300 \mathrm{~ns}$ were used to acquire nine spectra of bovine insulin b-chain that consisted of 100 laser shots at a mass resolution of 10,000 . The spectra are overlaid in Figure 5. The flight times for the first six isotopic ions in the bovine insulin b-chain spectra are tabulated and compared in the inset table. The table also contains average deviation in terms of time (nanoseconds) and parts per million. Note that the deviation is greatest for the low abundance ions.

The effect of pulse voltage and delay time on mass measurement accuracy of MALDI/DE-RTOF is illustrated by the data contained in Table 3. A sample spot was prepared by using a mixture of angiotensin II and the analytes listed in Table 3 . Mass spectra were taken from this spot at several pulse voltages and delay times. For the voltages and delays shown in Table 3, the mass resolution of each analyte is $>6500$. Each spectrum was calibrated by using the ACCA [2M + $\mathrm{H}]^{+}$and the angiotensin $\mathrm{II}[\mathrm{M}+\mathrm{H}]^{+}$ions.

Table 2. Mass measurement accuracy of MALDI/DE-RTOF with internal calibration

\begin{tabular}{|c|c|c|c|c|c|c|}
\hline \multirow[b]{2}{*}{ Spectra \# } & \multicolumn{2}{|c|}{ Melittin } & & \multicolumn{2}{|c|}{ Insulin b-chain } & \\
\hline & $\begin{array}{c}\text { Flight time } \\
\text { (ns) }\end{array}$ & $m / z$ & & $\begin{array}{c}\text { Flight time } \\
\text { (ns) }\end{array}$ & $m / z$ & \\
\hline 1 & 182179.23 & 2845.749 & & 201867.71 & 3494.600 & \\
\hline 2 & 182179.80 & 2845.789 & & 201868.79 & 3494.667 & \\
\hline 3 & 182180.41 & 2845.813 & & 201868.39 & 3494.659 & \\
\hline 4 & 182178.14 & 2845.755 & & 201868.27 & 3494.672 & \\
\hline 5 & 182179.03 & 2845.761 & & 201868.10 & 3494.636 & \\
\hline 6 & 182178.56 & 2845.743 & & 201867.19 & 3494.599 & \\
\hline 7 & 182179.58 & 2845.783 & & 201868.35 & 3494.652 & \\
\hline 8 & 182179.48 & 2845.764 & & 201868.21 & 3494.625 & \\
\hline 9 & 182178.49 & 2845.772 & & 201866.67 & 3494.622 & \\
\hline 10 & 182179.42 & 2845.793 & & 201868.86 & 3494.688 & \\
\hline Average & 182179.21 & 2845.772 & & 201868.05 & 3494.642 & \\
\hline Avg. deviation & 0.53 & 0.018 & & 0.52 & 0.026 & \\
\hline Avg. deviation (ppm) & 2.9 & 6.2 & & 2.6 & 7.3 & \\
\hline Actual $m / z$ & & & 2845.762 & & & 3494.651 \\
\hline Mass error & & & 0.010 & & & -0.009 \\
\hline Mass accuracy (ppm) & & & 3.6 & & & -2.6 \\
\hline
\end{tabular}




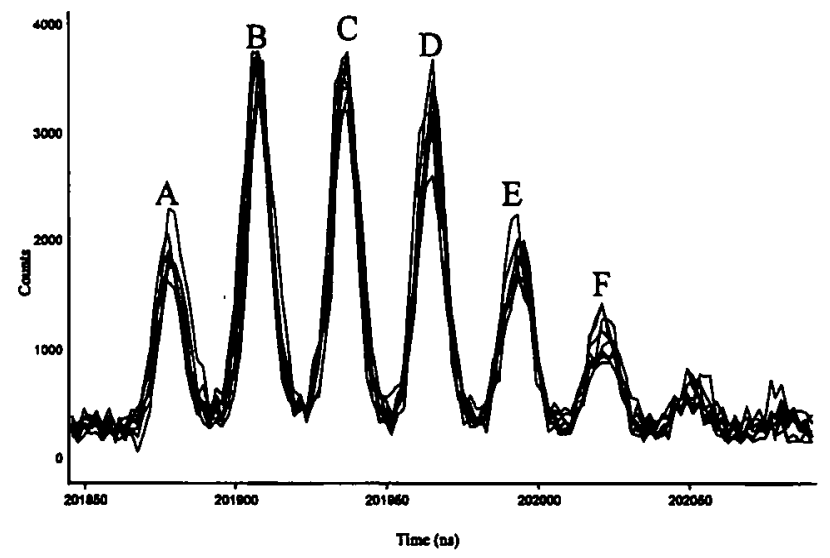

Flight time Reproducability of Bovine Insulin B-chain Isotopes

\begin{tabular}{|c|c|c|c|c|c|c|}
\hline & $\bar{A}$ & 8 & C & $\bar{D}$ & E & $\bar{F}$ \\
\hline & $\begin{array}{l}\text { Alght time } \\
\text { (ns) }\end{array}$ & $\begin{array}{l}\text { Night time } \\
\text { (ns) }\end{array}$ & $\begin{array}{l}\text { flight time } \\
\text { (ns) }\end{array}$ & $\begin{array}{l}\text { flight time } \\
\text { (ns) }\end{array}$ & $\begin{array}{l}\text { might time } \\
\text { (na) }\end{array}$ & $\begin{array}{l}\text { filght time } \\
\text { (ns) }\end{array}$ \\
\hline Spectra 1 & 201879.23 & 201007.89 & 201938.90 & 201965.49 & 201994.88 & 202022.60 \\
\hline Spectra 2 & 201877.64 & 201907.72 & 201936.30 & 201965.47 & 201994.38 & 202021.28 \\
\hline Spectra 3 & 201877.51 & 201806.80 & 201936.35 & 201964.94 & 201992.61 & 202022.73 \\
\hline Spectra 4 & 201878.21 & 201807.08 & 201935.40 & 201965.18 & 201993.35 & 202022.42 \\
\hline Spectra 5 & 201876.93 & 201908.81 & 201935.55 & 201964.82 & 201993.42 & 202021.15 \\
\hline Spectra 6 & 201878.68 & 201806.80 & 204836.31 & 201964.16 & 201893.35 & 202020.92 \\
\hline Spectra 7 & 201878.92 & 201908.32 & 201937.41 & 201965.79 & 201993.85 & 202023.29 \\
\hline Spectra 8 & 201878.61 & 201906.23 & 201935.20 & 201963.13 & 201992.37 & 202021.49 \\
\hline Spectra $\theta$ & 201878.13 & 201907.39 & 201935.95 & 201964.88 & 201993.88 & 202021.58 \\
\hline Average & 201878.32 & 201907.28 & 201938.15 & 201984.87 & 201993.58 & 202021.84 \\
\hline $\begin{array}{l}\text { Average } \\
\text { deviation }\end{array}$ & 0.66 & 0.51 & 0.56 & 0.56 & 0.62 & 0.73 \\
\hline $\begin{array}{l}\text { Average } \\
\text { deviation } \\
\text { (ppm) }\end{array}$ & 3.3 & 2.5 & 2.8 & 2.8 & 3.1 & 3.6 \\
\hline
\end{tabular}

Figure 5. An overlay of nine MALDI/DE-RTOF mass spectra of bovine insulin b-chain that shows the $[\mathrm{M}+\mathrm{H}]^{+}$ions acquired at a total acceleration of $20 \mathrm{kV}$, a pulse voltage of $6 \mathrm{kV}$, and a 300-ns delay time. A, B, C, D, E, and F denote the first six isotope peaks.

A sample spot that contained a three component peptide mixture was prepared and the MALDI/DERTOF mass spectrum was collected (Figure 6). Protonated and cationized species of each analyte are present. The insets in the figures contain expanded regions for the $[\mathrm{M}+\mathrm{H}]^{+}$ions of each analyte. The pulse voltage $(4 \mathrm{kV})$ and delay time (300 ns) used to acquire the spectrum shown in Figure 6 provide sufficient resolution $(m / \Delta m>6500)$ over a broad range of mass-to-charge ratio values (1-4 ku). The spectrum shown in Figure 6 was calibrated by using the $[\mathrm{M}+$ $\mathrm{Na}^{+}$and $\left[2 \mathrm{M}+\mathrm{H}^{+}\right.$ions of ACCA. The mass accuracies for angiotensin II, $\alpha$-melanocyte, and bovine insulin b-chain are 1,6 , and $-4 \mathrm{ppm}$, respectively.

Table 3. Mass accuracy in parts per million at different delay times and pulse voltages

\begin{tabular}{lccc}
\hline & \multicolumn{3}{c}{ Pulse voltage } \\
\cline { 2 - 4 } & $\begin{array}{c}6 \mathrm{kV} \\
150-\mathrm{ns} \\
\text { delay }\end{array}$ & $\begin{array}{c}4 \mathrm{kV} \\
450-\mathrm{ns} \\
\text { delay }\end{array}$ & $\begin{array}{c}45 \mathrm{kV} \\
650-\mathrm{ns} \\
\text { delay }\end{array}$ \\
\hline \hline Angiotensin I & 6 & 3 & 5 \\
Substance P & -4 & 8 & 6 \\
$\alpha$-Melanocyte & 1 & -4 & 2 \\
Melittin & 3 & 0 & -7 \\
\hline
\end{tabular}

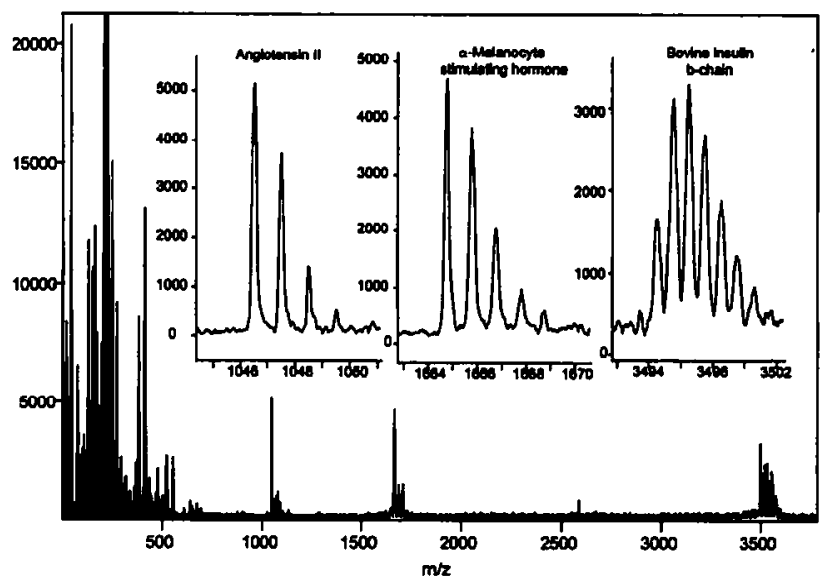

Figure 6. MALDI/DE-RTOF mass spectrum of a mixture of angiotensin II, $\alpha$-melanocyte, and bovine insulin b-chain acquired at a total acceleration of $20 \mathrm{kV}$, a pulse voltage of $4 \mathrm{kV}$, and a 300-ns delay time. The insets show the regions of the spectrum that contain only the protonated molecule of each analyte.

\section{Discussion}

On the basis of the previously reported work and the work reported here, it is clear that delayed extraction (DE) greatly improves the mass resolution for MALDITOF and MALDI-RTOF. Furthermore, the results from MALDI/DE-TOF reported by Whittal and Li [14] and Brown and Lennon [17] and the MALDI/DE-RTOF reported herein show substantial improvement in mass measurement accuracy over the $0.01 \%$ obtained using MALDI-TOF. For example, external calibration for the synthetic peptide shown in Figure 2 yields mass measurement accuracy of 8 and $10 \mathrm{ppm}$ for the $[\mathrm{M}+\mathrm{H}]^{+}$ and the $[\mathrm{M}+2 \mathrm{H}]^{2+}$ ions, respectively. The data contained in Table 1 demonstrate that the average mass measurement accuracy of MALDI/DE-RTOF by using external calibration is $\sim 13 \mathrm{ppm}$.

In this study we evaluated both external and internal calibration because we frequently observe signal suppression by one analyte in the presence of another; therefore, it is sometimes necessary to perform the analysis without the aid of an internal calibrant. In the evaluation of external calibration it is important to know the reproducibility of flight times between different sample spots. We find that the average deviation in flight time for an analyte is approximately $5 \mathrm{ppm}$ from spot to spot (Figure 4). A 5-ppm deviation in the flight time corresponds to a mass measurement accuracy of $10 \mathrm{ppm}$. Thus, we expect the mass measurement accuracy limit of externally calibrated MALDI spectra to be on the order of $10 \mathrm{ppm}$. This limit has several contributors, one of which is the short term drift of the high voltage power supplies (rated for 10-ppm short term drift). In addition, if there is a significant time lapse between the acquisition of the analyte and calibrant data, additional errors could arise from long term drift of the high voltage power supplies (100 ppm/day). We observe significant flight 
time shifts due to drift in the power supplies if they are not warmed up an hour prior to data acquisition. Sample surface inhomogeneity is another probable source of error. Although the sample preparation technique used in this study provides a homogeneous area of matrix-analyte crystals, variations in the sample thickness due to surface irregularities vary the distance between the plate and the first extraction grid and can result in variations of ion flight times. A variation of only $10 \mu \mathrm{m}$ in the sample surface produces a flight time shift of $1 \mathrm{~ns}$, which corresponds to a deviation of $5 \mathrm{ppm}$ for a $200-\mu \mathrm{s}$ flight time, for example, bovine insulin b-chain.

The reproducibility of ion flight times taken from the same sample spot (Figure 3, Table 2) is better than those taken from different sample spots. The major source of deviation in ion flight times formed from the same sample spot is jitter in the photodiode used to synchronize ion formation with data acquisition and the pulsed high voltage switch. We attempted to evaluate the jitter associated with each individual event, but the measurements were inconclusive because it is the collective effect that is important. We conclude that average deviations in flight time of only 2-3 ppm clearly show that the error associated with jitter in the trigger circuit is small, at least $<2-3 \mathrm{ppm}$.

Ultimately the limiting factor in mass measurement accuracy is our ability to centroid narrow peaks with a $500-\mathrm{MHz}$ digitizer (500 MS/s). For example, the full width at half maximum of the bovine insulin b-chain $[\mathrm{M}+\mathrm{H}]^{+}$ion acquired at a resolution of 6500 is $\sim 15 \mathrm{~ns}$, which provides only 7-8 data points from which to centroid the peak profile. The data in Table 2 clearly illustrate the ability to accurately centroid these peaks; they have deviations of only $2.6 \mathrm{ppm}$. If the peak profile and the peak centroid determination are limited by the number of data points, we would expect the deviations in flight time to significantly increase as the peaks become narrower. To evaluate the effects of fewer data points across the peak profile, spectra of bovine insulin b-chain $[\mathrm{M}+\mathrm{H}]^{+}$ions were acquired at a resolution of 10,000 (Figure 5). The peak width (FWHM) for bovine insulin b-chain $[\mathrm{M}+\mathrm{H}]^{+}$ions at 10,000 resolving power is $10 \mathrm{~ns}$, which provides only five data points to centroid the peak profile. The data contained in Figure 5 illustrate the high degree of reproducibility of the peak profile $(0.61 \mathrm{~ns}, 3 \mathrm{ppm})$. Note that the observed flight time deviation increases as the signal-to-noise ratios decreases. Thus, accurate mass measurements ultimately will be limited by the signal-to-noise ratio for the ion signal.

A clear advantage in terms of mass measurement accuracy of the long flight tube of the Voyager Elite XL is the long flight times of the ions. The effects of deviations in sample homogeneity and jitter impact the reproducibility of the spectra half as much as an instrument of half the length. A further disadvantage of a shorter flight tube is that it necessitates the use of a faster digitizer. One might argue that the same effect could be observed on a smaller instrument by lowering the ion acceleration voltage, which thus increases the flight time; however, we observe that both collection efficiency and resolution decrease as the acceleration voltage decreases. It is possible to offset the observed signal decrease as the acceleration voltage is decreased by increasing magnitude of the voltage applied to the WIG; however, increased WIG voltage degrades the mass resolution.

MALDI mass spectra often are calibrated by using two internal standards to bracket the analyte ion of interest, which allows the calibration curve to be interpolated over the mass-to-charge range of the analyte(s). This interpolation typically yields more accurate results than extrapolation of a calibration curve to high mass-to-charge ratio values. Deviations caused by extrapolation of the calibration curve presumably are due to ions of different masses that have different kinetic energies. There are two sources of mass-dependent kinetic energies to be considered: (1) Beavis and Chait reported that MALDI-formed ions were ejected from the surface with a velocity $(v)$ of $\sim 750 \mathrm{~m} / \mathrm{s}$ [3]; thus $v_{\text {total }}=v_{\text {inital }}+v_{E \text {-field }}$ and as the mass-to-charge ratio of the ion increases, a greater fraction of $v_{\text {total }}$ is due to $v_{\text {initial }}$. (2) Two studies reported that MALDIformed ions have lower velocities than expected based on the acceleration voltage applied to the ion source [2, 21]. Lower ion velocities or energy deficits could arise if the ions undergo collisions with desorbed neutrals or if ion formation occurs above the sample surface. In the $\mathrm{DE}$ experiment that used the overlayer sample preparation, we did not observe an effect for either process. The mass measurement accuracy shown in Table 2 is the result of a calibration curve generated by using $\alpha$-melanocyte $(m / z 1664.801)$ and a matrix ion $(\mathrm{m} / z$ 379.093) that is extrapolated 1200 mass units in the case of melittin and nearly 2000 mass units for bovine insulin b-chain $[\mathrm{M}+\mathrm{H}]^{+}$ion. The accuracy of the extrapolated calibration curves clearly demonstrates the ability of the reflectron to compensate for the mass-dependent energy component of the MALDI ions formed by using delayed extraction, even though the delay times and pulse voltages are not optimized for each individual analyte. The ability of the reflectron to minimize the mass-dependent energy is further reinforced by the data shown in Table 3. For example, the mass measurement accuracy changes very little as the pulse voltages and delay times are varied over a range in which good resolution is obtained for the analytes. Thus, the parameters of delayed extraction do not effect the mass measurement accuracy directly, but rather indirectly by effecting the mass resolution. Optimization of the pulse voltage and delay time for each mass-to-charge ratio value results in a mass resolution of 10,000-15,000 over a narrow mass-to-charge ratio range (Figure 1). The spectrum contained in Figure 6 illustrates the fact that the pulse voltage and delay time can be adjusted to give good mass resolution (> 6500) and low parts per million mass measure- 
ment accuracy over a broad mass-to-charge ratio range (1-4 ku). This is observed also in Figure 2, where good mass accuracy is obtained for both the $[\mathrm{M}+\mathrm{H}]^{+}$and the $[\mathrm{M}+2 \mathrm{H}]^{2+}$ ions. The mass range over which we obtain good resolution and good mass measurement accuracy is much smaller ( $<1 \mathrm{ku}$ ) when DE is used in the linear mode ([22] and Barbacci, D. C.; Edmonson, R. D.; Russell, D. H., unpublished manuscript).

It is important to note that extrapolation of a calibration curve derived solely from matrix ions (Figure 5) is highly dependent on the quality of the matrix spectrum. If the peak shapes for matrix ions are poor (saturated signal, unresolved detector ringing, etc.), a calibration curve generated by using only matrix ions is not accurate when extrapolated to high mass-tocharge ratio values $(3500 \mathrm{u})$. An error of only $100 \mathrm{ps}$ in centroiding the matrix peak in such a case results in a 5-ppm shift at the measured mass-to-charge ratio of bovine insulin b-chain $[\mathrm{M}+\mathrm{H}]^{+}$ions. Thus, it is better to calibrate by using a matrix ion and one internal standard. If the mass measurement requires accuracy at the 5-ppm level, it is necessary to calibrate by using a matrix ion and a single internal standard; however, if the desired accuracy is in the $10^{-15} \mathrm{ppm}$ range, external calibration is sufficient.

The different sample preparation methods commonly used effect many observables in the MALDI experiment, but most of the observed effects are related to the homogeneity of the matrix-analyte solid. For example, the ion formation thresholds depend upon the matrix used and the sizes of the matrix crystals formed. The abundance of fragment ions observed as "prompt fragments" or metastable ions also depends upon the sample preparation method used ([23-25] and Edmondson, R. D.; Barbacci, D. C.; Russell, D. H., unpublished manuscript). Likewise, the mass resolution and mass measurement accuracy depend upon the sample preparation methods. It appears that the overlayer sample preparation method used in this study provides a large area of uniform microcrystals with no visible "islands" that result in "hot spots." A detailed evaluation of the sample preparation used in this study will be discussed elsewhere (Edmondson, R. D.; Barbacci, D. C.; Russell, D. H., unpublished manuscript).

\section{Conclusions}

The data reported herein clearly show the mass measurement accuracy of MALDI/DE-RTOF by using external calibration is in the range of $10-15 \mathrm{ppm}$, and by using internal calibration the mass measurement accuracy is better than $5 \mathrm{ppm}$. Although the very best resolution $(10,000-15,000)$ is obtained by "tuning" the $\mathrm{DE}$ parameters over a narrow mass-to-charge ratio range, good mass measurement accuracy (5 ppm) is obtained by tuning the DE parameters to give good resolution $(m / \Delta m=6500)$ over a broad mass-tocharge ratio range $(1000-4000 \mathrm{u})$. In addition, the
MALDI/DE-RTOF calibration curves, both internal and external, are very linear [ $t$ versus $\left.(m / z)^{1 / 2}\right]$; they remain accurate even when extrapolated several thousand mass units.

\section{Acknowledgments}

This work was supported by a grant from the Urited States Department of Energy, Division of Chemical Sciences, Office of Basic Energy Science. Some of the equipment used in this research was purchased by funds provided by the National Science Foundation (CHE-9223629). The funds for the purchase of the Voyager Elite XL were provided by the College of Science, Texas A \& M University. The synthetic peptide was provided by M. Scholtz, College of Medical Sciences, Texas A \& M University.

\section{References}

1. Karas, M.; Bachmann, D.; Bahr, U.; Hillenkamp, F. Int. J. Mass Spectrom. Ion Processes 1987, 78, 53.

2. Zhou, J.; Ens, W.; Standing, K. G.; Verentchikov, A. Rapid Commun. Mass Spectrom. 1992, 6, 671-678.

3. Beavis, R. C.; Chait, B. T. Chem. Phys. Lett. 1991, 5, 479-484.

4. Pan, Y.; Cotter, R. J. Org. Mass Spectrom. 1992, 27, 3-8.

5. Mamyrin, B. A.; Karatajev, V. J.; Shmikk, D. V.; Zagulin, V. A. Sov. Phys.-JETP 1973, 37, 45-48.

6. Spengler, B.; Kirsch, D.; Kaufmann, R. Rapid Commun. Mass Spectrom. 1991, 5, 198-202.

7. Beavis, R. C.; Chait, B. T. Anal. Chem. 1990, 62, 1836-1840.

8. Solouki, T.; Gillig, K. J.; Russell, D. H. Rapid Commun. Mass Spectrom. 1994, 8, 26-31.

9. Zubarev, R. A.; Demirev, P. A.; Hakansson, P.; Sundqvist, B. U. R. Anal. Chem. 1995, 67, 3793-3798.

10. Wiley, W. C.; McLaren, I. H. Rev. Sci. Instrum. 1955, 26, 1150-1157.

11. Colby, S. M.; King, T. B.; Reilly, J. P. Rapid Commun. Mass Spectrom. 1994, 8, 865-868.

12. Brown, R. S.; Lennon, J. J. Anal. Chem. 1995, 67, 1998-2003.

13. Vestal, M. L.; Juhasz, P.; Martin, S. A. Rapid Commun. Mass Spectrom. 1995, 9, 1044-1050.

14. Whittal, R. M.; Li, L. Anal. Chem. 1995, 67, 1950-1954.

15. Li, Y.; McIver, R. T.; Hunter, R. L. Anal. Chem. 1994, 66. 2077-2083.

16. Pasa-Tolic, L.; Huang, Y.; Guan, S.; Kim, H. S.; Marshall, A. G. J. Mass Spectrom. 1995, 30, 825-833.

17. Brown, R. S.; Lennon, J. J. Anal. Chem. 1995, 67, 3990-3999.

18. Beavis, R. C. Anal. Chem. 1993, 65, 496-497.

19. Senko, M. W.; Beu, S. C.; McLafferty, F. W. J. Am. Soc. Mass Spectrom. 1995, 6, 229-233.

20. Geno, P.; Macfarlane, R. Int. J. Mass Spectrom. Ion Processes 1986, 74, 43-57.

21. Kinsel, G. R.; Edmondson, R. D.; Russell D. H. Proceedings of the 43rd ASMS Conference on Mass Spectrometry and Allied Topics; Atlanta, GA, May 21-26, 1995.

22. Colby, S. M.; Reilly, J. P. Anal. Chem. 1996, 68, 1419-1428.

23. Kinsel, G. R.; Preston, L. M.; Russell, D. H. Biol. Mass Spectrom. 1994, 23, 205-211.

24. Sumner, L. W.; Edmondson, R. D.; Gimon-Kinsel, M. E.; Hammon, R.; Sulikowski, G.; Russell, D. H. Proceedings of the 43rd ASMS Conference on Mass Spectrometry and Allied Topics; Atlanta, GA, May 21-26, 1995; p 1250.

25. Edmondson, R. D.; Russell, D. H. Proceedings of the 43rd ASMS Conference on Mass Spectrometry and Allied Topics; Atlanta, GA, May 21-26, 1995; p 686. 